\title{
Diagnostic and therapeutic role of laparoscopy in the Current Fertility Practice
}

\author{
Zakaria $\mathrm{R}^{1}$, Dewan $\mathrm{F}^{2}$, Sultana $\mathrm{Z}^{3}$, Ghani $\mathrm{A}^{4}$
}

\begin{abstract}
Background: Laparoscopy is perceived as a minimally invasive surgical technique that both provides a panoramic \& magnified view of the pelvic organs and allows surgery at the time of diagnosis. Laparoscopy has became an integral part of gynaecologic surgery for the diagnosis and treatment of abdominal and pelvic disorders of the Female reproductive organs. Endoscopic reproductive surgery intended to improve fertility many includes surgery of the uterus, ovaries, pelvic peritoneum and the fallopian tubes. Aim: To determine the best treatment for infertility. Method: The prospective study was carried out among the infertile women during the period of July 2014 to December 2014, in Shaheed Suhrawardi Medical College Hospital. Fifty women identified with Infertility both primary and secondary with the criteria of menstrual cycles with duration of 25-45 days were selected. Semen analysis was considered to be normal. Result: Fifty women underwent diagnostic \& therapeutic laparoscopy between the period of July 2014 to December 2014. Among them 20 suffered from primary subfertility \& 30 from secondary subfertility. In primary subfertility 12 patients were diagnosed as poiycystic ovarian disease \& 7 patients diagnosed as minimal to moderate endometriosis 1 patient diagnosed as pelvic inflammatory disease. In secondary subfertility 15 patients diagnosed as pelvic inflammatory disease, 12 patients with endometriosis \& 03 patients as poiycystic ovarian disease. Therapeutic intervention were done in almost all cases of subfertility. Conclusion: The routine use of diagnostic laparoscopy for the evaluation of all case of female infertility is currently under debate. Current evidence indicates that the surgical treatment of minimal or mild endometriosis increase the spontaneous pregnancy rate in Infertility women.[J Shaheed Suhrawardy Med Coll, 2013;5(2):73-75]
\end{abstract}

Keywords: Subfertility, Endometroisis, Pelvic inflammatory disease, Poiycystic ovarian disease.

Received: August 2012; Revised: October 2013; Accepted: November 2013

\section{Introduction}

The role of diagnostic laparoscopy in current fertility practice is controversial. According to the American Fertility Society in 1992 and by the World Health Organization guidelines (Rowe et al. 1993) laparoscopy was the final diagnostic procedure of the female fertility workup $^{1}$. In 1997, Glatstein et al (1997) reported $89 \%$ of all reproductive endocrinologists in USA routinely performed a laparoscopy has been done in the diagnostic work-up of infertility ${ }^{2}$. However many authors have subsequently shown that laparoscopy in up to $70 \%$ of cases $^{3}$. Currently diagnostic laparoscopy is often bypassed by many Infertility specialists in favour of moving forward with assisted reproductive technology such as intrauterine insemination (IUI) or in vitro fertilization (IVF) as the procedure requires general anesthesia and can be associated with a low but potentially serious incidence of risks and complication. In this study we show that

1. Dr. Rooh-e Zakaria, Assistant Professor, Department of Obstetrics \& Gynaecology, Shaheed Suhrawardi Medical College Hospital, Dhaka, Bangladesh.

2. Prof. Dr. Farhana Dewan, Professor \& Head of the Department of Obstetrics \& Gynaecology, Shaheed Suhrawardi Medical College Hospital, Dhaka, Bangladesh.

3. Dr. Zobaida Sultana Susan, Junior Consultant, Department of Obstetrics \& Gynaecology, Shaheed Suhrawardi Medical College Hospital, Dhaka, Bangladesh.

4. Dr. Afroza Ghani, Assistant Professor, Department of Obstetrics \& Gynaecology, Shaheed Suhrawardi Medical College Hospital, Dhaka, Bangladesh.

Correspondence

Dr.Rooh-e-Zakaria, Assistant Professor, Department of Obstetrics \& Gynaecology, Shaheed Suhrawardi Medical College Hospital, Sher-E-Bangla Nagar, Dhaka, Bangladesh.roohezakaria@Rmail.com

Conflict of interest: None

Contributions by authors: All authors contributed from protocol preparation upto manuscript writing. 
diagnostic laparoscopy still has a role in infertility workup.

Disadvantage of diagnostic laparoscopy include the need for general aneasthesia, patients anxiety and the possibility of adhesion formation. In a large Finnish follow up study, the complication rate of diagnostic laparoscopy was 0.6 per 1000 procedure $^{4}$ (HaKKi-Siren et al. 1999). However, advantages include the possibility to perform both diagnostic and therapy at the same time and the opportunity to combine the laparoscopy with the hysteroscopic exploration of the uterine cavity with an endometrial biopsy all as part of day case surgery. Cardiff in 1995 reported that pathologic abnormalities are found in $21-68 \%$ with unexplained infertility at the time of diagnostic laparoscopy ${ }^{4}$. Badawi in 1999 and Comson in 2000 reproted similar findings ${ }^{5,6}$ In addition minor surgical procedures at the time of laparoscopy can enhance fertility in upto $20 \%$ of cases. It is well known that patency of the fallopian tubes by hysterosalpingogram does not rule out pelvic adhesion that can only be diagnosed by laparoscopy. Many couples are also interested in knowing the possible reason behind their infertility and diagnostic laparoscopy can shed some light on that. In our opinion, laparoscopy should be discussed with patients, especially if they have a normal ovarian reserve and are of young reproductive age. Laparoscopy can also benefit patients with unilateral or bilateral hydrosalpinx by removing the affected tubes and improving the chance of pregnancy with IVF or naturally in case of a unilateral hydorsalpix and a normal contralateral tube ${ }^{7.8}$. The prevalence of pelvic endometriosis is significantly higher in infertile women $(20-68 \%)$ compared to the general population ${ }^{9-11}$ ablation of endometriotic lesions with lysis of adhesion has been proven to be beneficial in infertile women compared to diagnostic laparoscopy alone ${ }^{12}$. in addition, the treatment of endometriosis by laparoscopy can enhance the chances of infertile women to conceive with IUI.

\section{Methodology}

The prospective study was carried out among the infertile women during the period of July 2014 to December 2014 at Shaheed Suhrawardi Medical College Hospital. Women identified with infertility both primary on secondary before laparoscopy satisfied the following criteria

1) Menstrual cycle with duration of 25-45 days

2) Semen analysis was considered normal

Pre-operative assessment of the patients including full history taking, thorough general examination and investigation for infertility work up \& anaesthesia fitness was done. Laparoscopy were done under general anaesthesia in combination with dye test.

\section{Results}

Fifty women underwent diagnostic \& therapeutic laparoscopy between the period of July 2014 to December 2014. Among them, 20 patients suffered from primary subfertility \& 30 patients suffered from secondary subfertility

In primary subfertility 12 patients diagnosed as polycystic ovarian disease \& 7 patients diagnosed as minimal to moderate lendometriosis. 1 patient diagnosed as PID.

In secondary subfertility 15 patients diagnosed as pelvic inflammatory disease, 12 patients diagnosed with endometriosis and 03 patients diagnosed as polycystric ovarian disease. Both the tube were patent (Dye test $+v e$ ) for 15 patients in primary subfertility and 03 patients in secondary subfertility .

Hydrosalpinx were present in 07 case. Adhesion were found in almost all cases of pelvic inflammatory diseases. Bilateral chocolate cyst were found in 2 cases of primary subfertility $\&$ in 3 case there unilateral chocolate cyst \& 2 cases there minimal endometriotic deposits. 08 patients with secondary subfertility \& 03 patients with unilateral chocolate cyst \& 01 patient with moderate adhesion due to endometriosis. Theraputic intervention were done in almost all cases of subfertility.

In poly cystic ovarian cases ovarian drilling were done with 4-6 puncture (according to no of cysts) with 4 watt energy (monopolar). Adhesiolysis were done as far as possible in almost all cases.

In PID cases the hydrosalpinx were punctured. In chocolate cyst the cysts -were ruptured $\&$ the cyst wall were removed.

Table I: Distribution of patients by age group $(n=50)$

\begin{tabular}{lcc}
\hline Age group & Frequency & Percentage (\%) \\
\hline $18-23$ & 12 & 24 \\
$24-29$ & 19 & 38 \\
$30-35$ & 13 & 26 \\
$36-40$ & 6 & 12 \\
\hline
\end{tabular}

Table: 2 Types of Infertility $(\mathbf{n}=\mathbf{2 0})$

\begin{tabular}{lcc}
\hline Type & Frequency & Percentage \\
\hline Primary subfertility & 20 & $40 \%$ \\
Secondary & 30 & $60 \%$ \\
\hline
\end{tabular}

Table: 3 Causes of primary subfertility $(n=20)$

\begin{tabular}{lc}
\hline PCOS & $12(24 \%)$ \\
Endometriosis & $7(14 \%)$ \\
PID & $1(2 \%)$ \\
\hline
\end{tabular}

Table: 4 Causes of secondary subfertility $(n=30)$

\begin{tabular}{lc}
\hline PCOS & $3(6 \%)$ \\
Endometriosis & $12(24 \%)$ \\
PID & $15(30 \%)$ \\
\hline
\end{tabular}


Table: 5 Treatment modalities (Laparoscopy)

\begin{tabular}{lcc}
\hline Disease & Findings & Treatmetn \\
\hline PID & Adhesion & Adhesiolysis \\
Endometriosis & Chocolate Cyst & Cystectomy \\
PCOS & Polycystic Ovary & Ovarian Drilling \\
\hline
\end{tabular}

Table: 6 Results of dye test (tubal patency test)

\begin{tabular}{lcc}
\hline Dye test & Positive (Patent) Negative (Blocked) \\
\hline $\begin{array}{l}\text { Primary Subfertility } \\
(\mathrm{n}=20)\end{array}$ & $15(30 \%)$ & $5(10 \%)$ \\
$\begin{array}{l}\text { Secondary Subfertility } \\
(\mathrm{n}=30)\end{array}$ & $3(6 \%)$ & $27(54 \%)$ \\
\hline
\end{tabular}

Table: 6 Results of dye test (tubal patency test)

\begin{tabular}{lcc}
\hline Dye test & Positive (Patent) Negative (Blocked) \\
\hline $\begin{array}{l}\text { Primary Subfertility } \\
(\mathrm{n}=20)\end{array}$ & $15(30 \%)$ & $5(10 \%)$ \\
$\begin{array}{l}\text { Secondary Subfertility } \\
(\mathrm{n}=30)\end{array}$ & $3(6 \%)$ & $27(54 \%)$ \\
\hline
\end{tabular}

\section{Discussion}

Recent advances in endoscopic surgical techniques and the increased sophistication of surgical instruments have offered new operative methods and techniques for the gynecologic surgeon ${ }^{1}$. Recent years have witnessed a marked increase in the number of gynecological endoscopic procedure performed, improvements in instruments.. The addition of a small video camera to the laparoscopic greatly enhanced the popularity of operative endoscopy because of the possibility of operative in a comfortable, upright position and using the magnification capabilities of the camera ${ }^{13}$ Currently, laparoscopy is perceived as a minimally invasive surgical technique that both provides a panoramic \& magnified view of the pelvic organs and allow surgery at that time of diagnosis and treatment of abdominal and pelvic disorder of the female reproductive organs. Endoscopic reproductive surgery intended to improve fertility many include surgery of the uterus, ovaries, pelvic peritoneus and the fallopian tubes. Diagnostic \& therapeutic role of laparoscopy in infertility is unquestionable. Endometriosis is a heterogenous disease with typical and atypical morphology and spuns a sprectum from single $1 \mathrm{~mm}$ peritoneal implant to $10 \mathrm{~cm}$ or larger endometrioses with cul-de xac obliteration $\mathrm{M}$ severe endometriosis is associated with pelvic adhesions and a distortion of pelvic anatomy leading to a possible mechanic or anatomic disturbance of fertility.
Endometriomas and drained, biopsied and vaporized by using a laser or electro surgery or removed in pieces. Certain other surgical procedures are also done laparoscopically such as salpingo ovariolysis is division of adhesions involving fallopian tube and ovary, salpingostomy is the refashioning of a distal tubal ostium for distal tubal occlusion and designed to keep the fallopian tube open.

\section{Conclusion}

The routine use of diagnostic laparoscopy for the evaluation of all case of female infertility is currently under debate . Current evidence indicates that the surgical treatment of minimal or mild endometriosis increase the spontaneous pregnancy rate in Infertile women.

\section{References}

1. Rowe PJ, Comhaire FH, Hargerave TB, Mahmoud AMA, WHO manual for the standardized investigation of the infertile couple. Cambridge University Press, Cambridge, UK (1993)

2. Glatstein IZ, Harlow BL, Hornstcin MD. Practice patterns among reproductive endocrinologists: The infertility evaluation. Fertile Steril 1997;67:443-51.

3. R.G. Forman, J.N. Robinson, Z. Mehta, D.H. Barlow Patient history as a simple predictor of pelvic pathology in subfertile women. Hum Reprod 1993;8:53-5.

4. Cundiff G, Carr BR, Marshburn PB, Infertile couples with a normal hysterosulpingogram. Relationship to clinical and laparoscopic findings. $\mathrm{J}$ Reprod Med 1995;40:19-24.

5. Al Badawi IA, Fluder MR, Bebbington MW. Diagnostic laparoscopy in infertile women with normal hysterosalpingogram. Reprod Med 1999;44:953-7.

6. Corson SL, Cheng A, Guthman JN. Laparoscopy in the normal infertile patient: a question revisited J am Assoc Gynecol Laparosc 2000;7:317-24. 7. Dechaud H, Daures JP, Arnal F, Jumcau C, Hedon B. Does previous salpingectomy improve implantation and pregnancy rates in patients with severe tubal factor infertility who are undergoing in vitro fertilization? A priot prospective randomized study. Fertile Steril 1998;69:1020-5.

8. Strandell A, Lindhard A, Waldenstrom U, Thorburn J, Janson PO. Hamberger L. Hydrosalpinx and IVF outcome: a prospective, randomized multicentre trial in Scandinavia on salpingectomy prior to IVF. Jum Reprod 1999;14:2762-9.

9. Houston DE, IMoller KL, Melton U, Selwyn BJ, Hardy RJ. Incidence of pelvic endometriosis in Rochester, Minnesota. 1970-1979. Epidemiology 1987:125:959-69.

10. Mahmood TA, Templeton A. Prevalence and genesis of endometriosis. Jum Reprod 1991; 6:544-9.

11. Templeton A, Morris JK, Parslow W. Factors that affect outcome of in-vitro fertilization

treatment. Lancet 1996; 348:1402-6.

12. Jacobson TZ, Barlow DH, Koninckx PR, Olive D, Farquhar C. Laparoscopic surgery. for subfertility associated with endometriosis (Cochrane review). In: The Cochrane Library. Issue 3. Chichcster, UK John Wiley \& Sons Ltd; 2004.

13. Verkauf BS, Myomectomy for infertility enhancement and preservation fertile steril 1992; 58:1-15.

14. Azarani A, Osias J, Berker B, Nezhat C. Endometiosis insights into its pathogenesis and treatment surg Techno lint 2004;12:178-81. 